\title{
Semi empirical models for drying of agricultural products by used structured artificial neural networks
}

\author{
Bessenyei, K.; Kurják, Z.; Beke, J. \\ Institute for Process Engineering. Szent István University, Gödöllő, Hungary \\ *E-mail of the corresponding author: bessenyei.kornel@gek.szie.hu
}

\begin{abstract}
We compared a semi empirical and an empirical model. The empirical model is a multilayer ANN. The semi empirical model is a custom multilayer ANN. It is a structured model, and we define the structure by hand before the training of the network. The influence of the neuron numbers on the accuracy of the models was also investigated by statistical approach.

We found that the custom multilayer ANNs developed like this, are suitable for modelling the drying process of agricultural materials. They also provide the ability to improve the applicability of the empirical models. Furthermore, the semi empirical model has a higher sensitivity on neuron number applied.
\end{abstract}

Keywords: drying; energetics; artificial neural network; semi-empirical model. 


\section{Introduction}

The complexity of the water removal process, and the inhomogenities of the process variables both in time and space make the modelling of the drying process of agricultural products difficult. The usability of theoretical models is limited due to the stochastic material properties. [4] In these cases, the application of empirical models is essential. [5] However, in the most cases we have theoretical information about the process. Applying this information to the model it will became semi-empirical and possibly improve the usability compared to the pure empirical. [3]

Artificial intelligence techniques are also found in drying. [6] They are used for process variable prediction [10][12][15], process control [9][11][13] or for soft sensors[14]. Artificial neural networks (ANN) are sucsefully applied on all these fiels on their own, or combined with other AI or conventional techniques. [8] The use of ANN requires attention and awareness as the structure, the training and data preparation have significant effect on usability of the model. [7]

Earlier we found that artificial neural networks (ANN) are suitable to model the drying of agricultural products. [1] They are empirical models, but can be turned into semi-empirical when we use the combination of smaller ANNs in a suitable structure instead of one unstructured model. [2]

It can happen that we have a priori information about the structure of the system (to be modelled), but don't have the data to train the subsequent ANNs that make up the semiempirical model as a combination of smaller individually trained networks. In this case a single, but structured ANN can be created. The ANN can be defined by hand. It is called a custom artificial neural network. It can have a structure like the combination of smaller ANNs have, but the model can be trained without having any data for the internal connection.

The statistical approach that we used earlier [1] can help us to find the optimal structure of the network. The models' performance can be highly influenced by the applied number of the layers, and the number of the neurons in the layers. There are structures and neuron numbers that will never ever provide us an accurate model. On the other hand, there are structures what provide us accurate model with a high probability.

This paper is about the semi-empirical ANN models made from structured analytical neural networks and it is looking for answers to the following questions: Is it possible to construct such a model? Is it possible to find an optimal model? Is it better than the pure empirical? 


\section{Materials and Methods}

The measurement data is taken from an experimental drying of tomato pomace in a laboratory dryer. The drying apparatus is a convective batch dryer. The dried material is placed into the drying chamber on perforated sheet metal trays in three layers. The psychrometric properties of the drying air are measured before and after each layer, the flow rate is measured with an orifice at the duct inlet. The water removal is measured with gravimetric method. For data acquisition five-minute sampling rate was used.
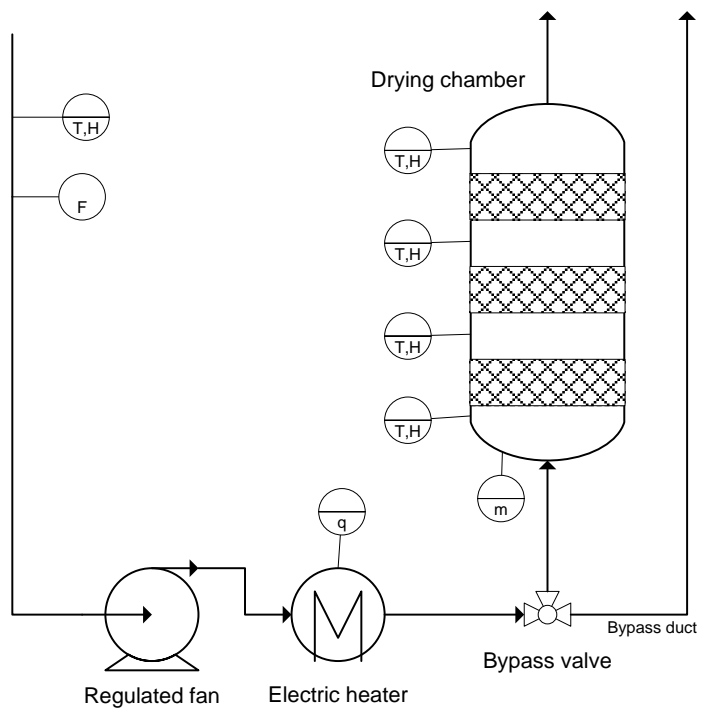

Fig. 1. Schematic of the dryer. (T-temperature, H-humidity, F-flow rate, m-mass, q-heat flow)

The wet-base moister content of the raw material was about $80 \%$ at the begining and $6 \%$ at the end of the drying experiment. The flowrate of the drying air was $370 \mathrm{~m}^{3} / \mathrm{h}$ and the temperature was set according to table $1.2 \mathrm{~kg}$ tomato pomes was put on each tray in a 40 mm thick layer.

Table 1. Temperature of the drying air

\begin{tabular}{cc}
\hline experiment & temperature $\left[{ }^{\circ} \mathbf{C}\right]$ \\
\hline 1 & 40 \\
3 & 110 \\
5 & 75 \\
6 & 55 \\
\hline
\end{tabular}

From the preliminary data analysis four data sets have been chosen for the models: experiment 1, 3, 5 and 6. From these data sets experiment 1 has systematic error, and not 
used for training the models but for test. It is required that the model shall approximate experiment 1 with a significantly higher error.

The first model constructed is purely empirical. According to the earlier works on this topic [1], a two-layer ANN has been chosen. Two such models were defined and we use the simpler one as it can be found on figure 2 below. The simplified model does not deal with the layers; it is like a one-layer dryer.

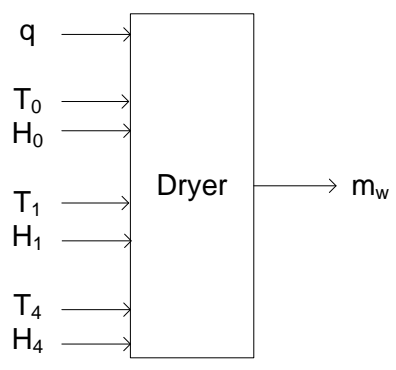

Fig. 2. Empirical model of deep bed dryer. ( $m_{w}$-water removal rate, $T_{0}$-ambient air temperature, $T_{1^{-}}$ air temperature at drying chamber inlet, $\mathrm{T}_{4}$-air temperature at drying chamber outlet, $\mathrm{H}_{0}$-ambient air humidity, $\mathrm{H}_{1}$-humidity at drying chamber inlet, $\mathrm{H}_{4}$-air humidity at drying chamber outlet)

The semi-empirical model is considering that the dryer consists of a burner and a drying chamber. The connection of the two components is the flow rate of the drying media.

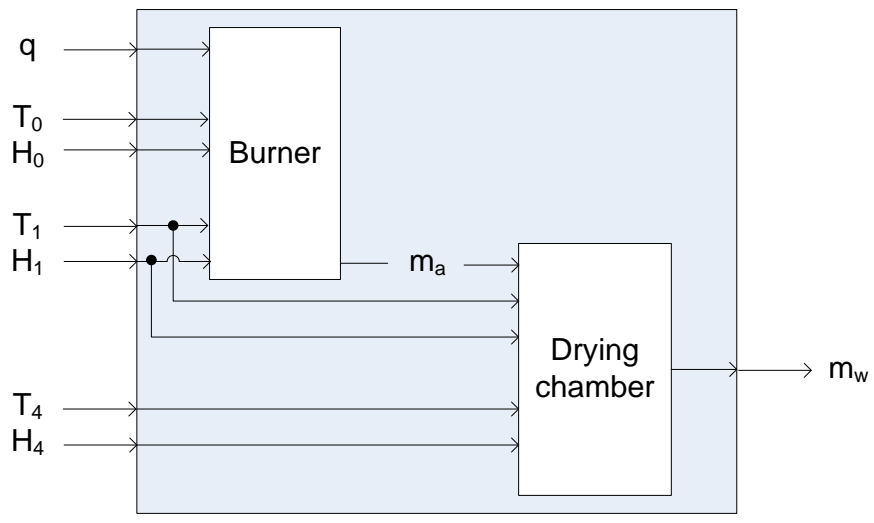

Fig. 3. Semi-empirical model of the deep bed dryer. ( $m_{a}$-flow rate of the drying air)

For the burner a one-layer ANN was used. For the drying chamber and the simplified empirical model, a two-layer structure was used. For the determination of the optimal neuron numbers in each layer the statistical approach was used. [1] We compared several networks with different neuron numbers in each. Table 2 contains the neuron numbers we used. 
After the training of the ANN models, we tested them. All the experiment data was given to the model inputs and the model output was compared to the measured data. For each simulation the cumulated squared error was calculated as the measure of model performance.

$$
\text { Cumulated_squared_error }=\sum(\text { measured }- \text { simulated })^{2}
$$

Table 2. Neuron numbers per layer

\begin{tabular}{ccc}
\hline model & layer & neurons \\
\hline empirical & $1^{\text {st }}$ & $1-15$ \\
& $2^{\text {nd }}$ & $1-15$ \\
\hline semi empirical & burner & $1-10$ \\
& drying chamber $1^{\text {st }}$ & $1-15$ \\
& ${\text { drying chamber } 2^{\text {nd }}}$ & $1-15$ \\
\hline
\end{tabular}

We also checked the correlation of the model output with the measured data. Figure 4 shows the algorithm of the whole process. Each structure was trained and tested 5 times. At the end, the results were shown in diagrams and the optimal model structure was chosen. The optimal model is the model with the smallest expected value and little standard deviation of cumulated squared error. The optimal models output should also have a good correlation with the measurement data.

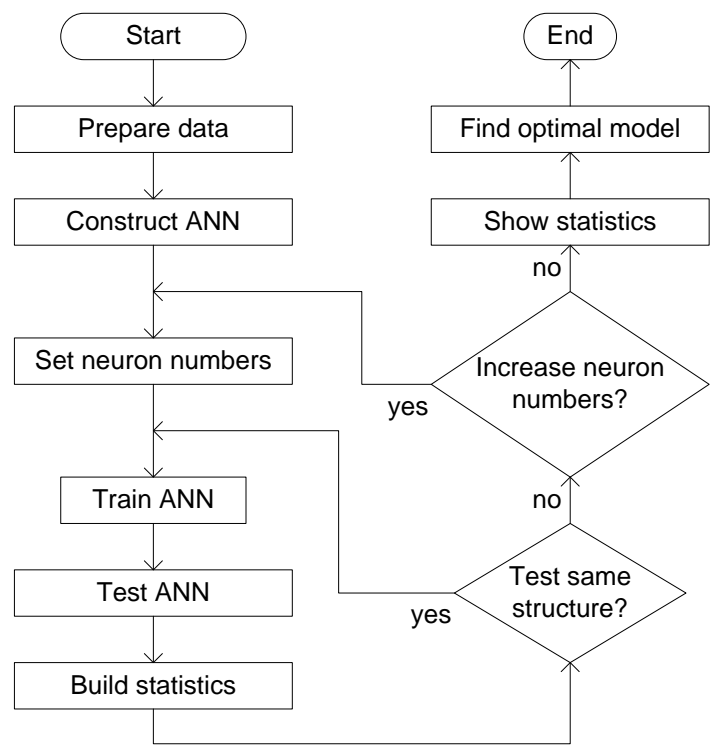

Fig. 4. Workflow of finding the optimal model 


\section{Results and Discussion}

The empirical model has 2 hidden layers. We used the method on Figure 4 to construct empirical models with different neuron numbers according to Table 2 . The results can be observed on Figure 5. The structure of the optimal empirical model and its performance is shown in Table 3.

Table 3. Optimal models

\begin{tabular}{cccc}
\hline model & layer & neurons & error \\
\hline empirical & $1^{\text {st }}$ & 7 & 0.862 \\
& $2^{\text {nd }}$ & 9 & \\
\hline semi empirical & burner & 1 & 2.6 \\
& ${\text { drying chamber } 1^{\text {st }}}^{\text {drying chamber } 2^{\text {nd }}}$ & 13 & \\
& dryin chan & 11 & \\
\hline
\end{tabular}
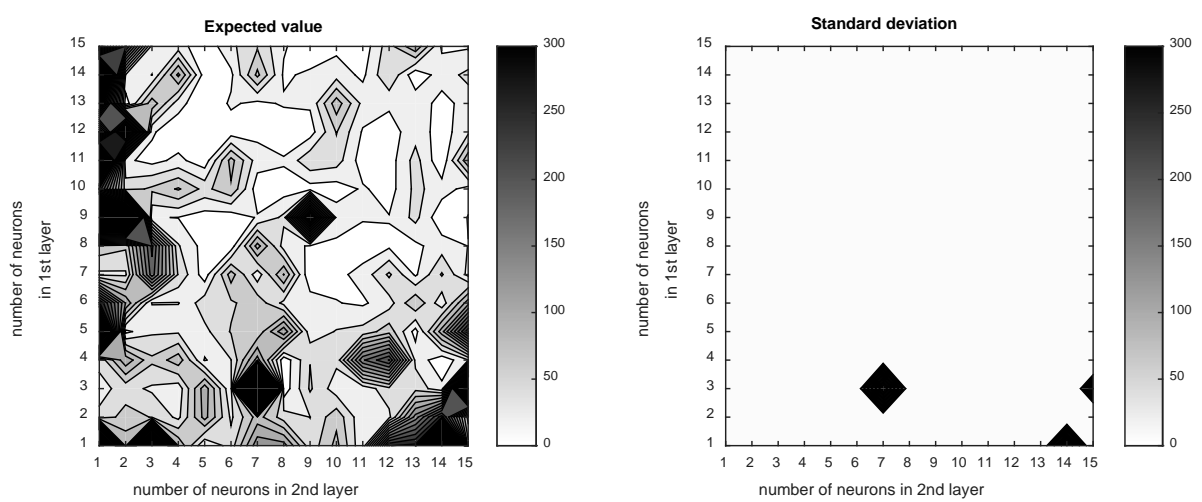

Fig. 5. Performance of the empirical model with different neuron numbers.

The semi empirical model always has 3 hidden layers: one for the burner and two for the drying chamber. We used the same method as for the empirical model thus on Figure 4. The results cannot be shown on one diagram as the 3 layers of neurons span a 3dimensional space rather than a 2-dimensional. But it is possible to show on several diagrams. We need as many diagrams, as many neurons we have in a chosen layer. We choose the burner layer, because it has less possible number of neurons than the others. This way we got the smallest number of diagrams. Figure 6 contains the optimal structure. The structure of the optimal semi empirical model and its performance can be found in Table 3. 

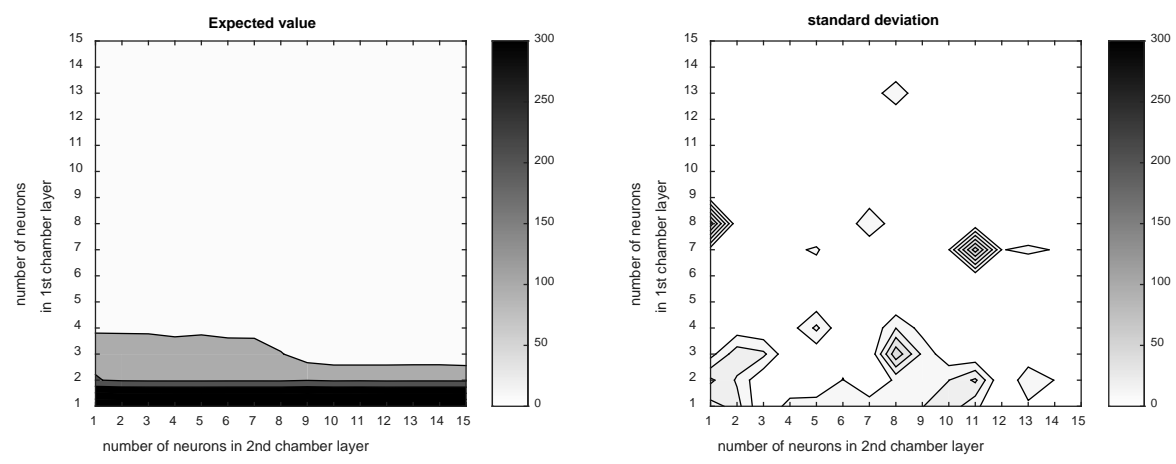

Fig. 6. Performance of the semi empirical model with a single neuron in the burner layer.

There are several near optimal structures on figure 6. On the other hand there are structures with significantly higher error what probably make the model unusable. If the burner has one neuron the $1^{\text {st }}$ chamber layer should have at least 3 or 4 neurons. The diagrams with higher neuron numbers for the burner show significantly higher error. Because of the limited space we did not include in this paper all the diagrams but they are very similar to Figure 7 in the aspect that the model with all those neuron numbers has significantly higher error.
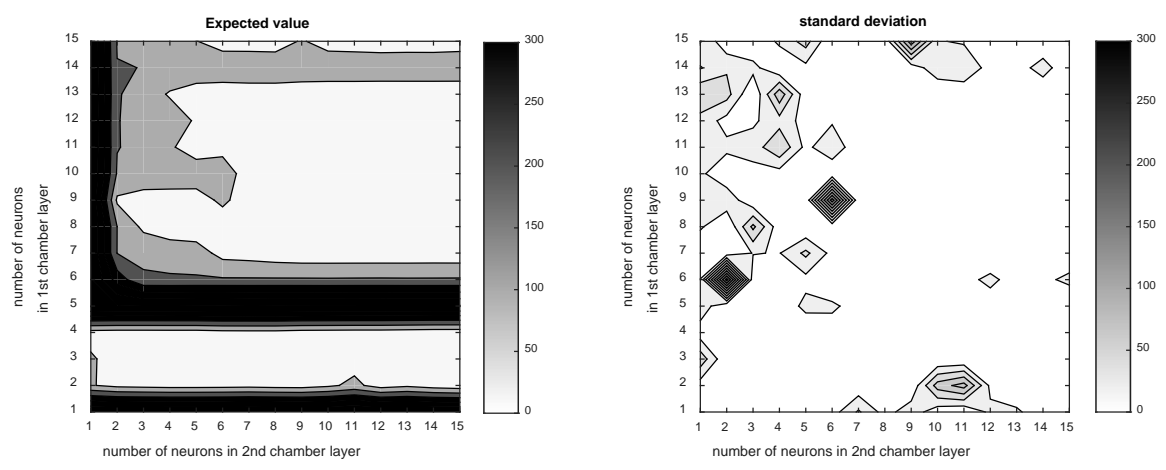

Fig. 7. Performance of the semi empirical model with 2 neuron in the burner layer.

\section{Conclusions}

Empirical and semi empirical models both approximated the measurement data with acceptable error.

It was possible to construct a semi empirical model with a custom ANN that is suitable for modelling the drying process of tomato pomace.

There are significant differences in model performance depending on the model structure and the number of neurons used in its layers. 
The statistical method we used can highlight the unusable and potentially usable structures.

With the statistical method we used it was possible to find the optimal model structure.

\section{References}

[1] Bessenyei, K.; Kurják Z.; Deákvári, J.; Beke J. Modelling of tomato pomace drying process with artificial neural network. In Proceedings of $5^{\text {th }}$ European Drying Conference, Paris, France, October 2-5, 2013.

[2] Bessenyei, K.; Kurják Z.; Beke J. Semi empirical models with artificial neural network. In Proceedings of $6^{\text {th }}$ European Drying Conference, Budapest, Hungary, October 21-23, 2015.

[3] Cubillos, F. A.; Wyhmeister, E.; Acuna, G.; Alvarez, P. I. Rotary dryer control using a gre-box neural model scheme. Drying Technology 2011, 29, 1820-1827.

[4] Ghaderi, A.; Abbasi, S.; Motevali, A.; Minaei, S. Comparison of mathematical models and artificial neural networks for prediction of drying kinetics of mushroom in microwave-vacuum drier. Chemical Industry \& Chemical Engineering Quarterly 2012, 18 (2), 283-293.

[5] Tohidi, M.; Sadeghi, M.; Mousavi, S. R.; Mireei, S. A. Artificial neural network modeling of process and product indices in deep bed drying of rough rice. Turkish journal of agriculture and forestry 2012, 36(6), 738-748.

[6] Martynenko, A. Artificial intelligence: Is it a good fit for drying? Drying Technology 2018, 36(8), 891-892.

[7] Farkas, I. Use of Artificial Intelligence for the Modelling of Drying Processes. Drying Technology 2013, 31, 848-855.

[8] Aghbashlo, M.; Hosseinpour, S.; Mujumdar, A. S. Application of Artificial Neural Networks (ANNs) in 175 Drying Technology: A Comprehensive Review. Drying Technology 2015, 33, 1397-1462.

[9] Nakamura, G.; Bentes, F.; Freire, J. Control of the Moisture Content of Milk Powder Produced in a Spouted Bed Dryer Using a Grey-Box Inferential Controller. Drying Technology 2015, 33(15-16), 1920-1928.

[10] Martínez-Martínez, V.; Gomez-Gil, J.; Stombaugh, T. S.; Montross, M. D.; Aguiar, J. M. Moisture Content Prediction in the Switchgrass (Panicum virgatum) Drying Process Using Artificial Neural Networks, Drying Technology 2015, 33:14, 1708-1719.

[11] Jianshuo Li; Qingyu Xiong; Kai Wang; Xin Shi; Shan Liang; Min Gao. Temperature control during microwave heating process by sliding mode neural network. Drying Technology 2016, 34(14), 1708-1719.

[12] Prerna Khawas; Kshirod Kumar Dash; Arup Jyoti Das; Sankar Chandra Deka. Modeling and optimization of the process parameters in vacuum drying of culinary banana (Musa ABB) slices by application of artificial neural network and genetic algorithm. Drying Technology 2016, 34(4), 491-503.

[13] Jianshuo Li; Qingyu Xiong; Kai Wang; Xin Shi; Shan Liang. A recurrent self-evolving fuzzy neural network predictive control for microwave drying process. Drying Technology 2016, 34(12), 1434-1444.

[14] Mansoureh Mozaffari; Asghar Mahmoudi; Kaveh Mollazade; Bahareh Jamshidi. Low-cost optical approach for noncontact predicting moisture content of apple slices during hot air drying. Drying Technology 2017, 35(12), 1530-1542.

[15] Ergün, A.; Ceylan, I.; Acar, B.; Erkaymaz, H. Energy-exergy-ANN analyses of solar-assisted fluidized bed dryer. Drying Technology 2017, 35(14), 1711-1720. 